\title{
Video Article \\ Remote Magnetic Navigation for Accurate, Real-time Catheter Positioning and Ablation in Cardiac Electrophysiology Procedures
}

\author{
David Filgueiras-Rama ${ }^{1}$, Alejandro Estrada ${ }^{1}$, Josh Shachar ${ }^{2}$, Sergio Castrejón ${ }^{1}$, David Doiny ${ }^{1}$, Marta Ortega ${ }^{1}$, Eli Gang ${ }^{3}$, José L. Merino ${ }^{1}$ \\ ${ }^{1}$ Cardiology, Robotic Cardiac Electrophysiology and Arrhythmia Unit, La Paz University Hospital \\ ${ }^{2}$ Magnetecs Corp. \\ ${ }^{3}$ Cardiology, Geffen School of Medicine at UCLA Los Angeles
}

Correspondence to: José L. Merino at jlmerino@arritmias.net

URL: https://www.jove.com/video/3658

DOI: doi:10.3791/3658

Keywords: Medicine, Issue 74, Anatomy, Physiology, Biomedical Engineering, Surgery, Cardiology, catheter ablation, remote navigation, magnetic, robotic, catheter, positioning, electrophysiology, clinical techniques

Date Published: 4/21/2013

Citation: Filgueiras-Rama, D., Estrada, A., Shachar, J., Castrejón, S., Doiny, D., Ortega, M., Gang, E., Merino, J.L. Remote Magnetic Navigation for Accurate, Real-time Catheter Positioning and Ablation in Cardiac Electrophysiology Procedures. J. Vis. Exp. (74), e3658, doi:10.3791/3658 (2013).

\section{Abstract}

New remote navigation systems have been developed to improve current limitations of conventional manually guided catheter ablation in complex cardiac substrates such as left atrial flutter. This protocol describes all the clinical and invasive interventional steps performed during a human electrophysiological study and ablation to assess the accuracy, safety and real-time navigation of the Catheter Guidance, Control and Imaging (CGCl) system. Patients who underwent ablation of a right or left atrium flutter substrate were included. Specifically, data from three left atrial flutter and two counterclockwise right atrial flutter procedures are shown in this report. One representative left atrial flutter procedure is shown in the movie. This system is based on eight coil-core electromagnets, which generate a dynamic magnetic field focused on the heart. Remote navigation by rapid changes ( $\mathrm{msec}$ ) in the magnetic field magnitude and a very flexible magnetized catheter allow real-time closed-loop integration and accurate, stable positioning and ablation of the arrhythmogenic substrate.

\section{Video Link}

The video component of this article can be found at https://www.jove.com/video/3658/

\section{Introduction}

Catheter ablation of cardiac arrhythmias has become an effective treatment for different types of cardiac arrhythmias. ${ }^{1,2}$ Antiarrhythmic drugs have limited efficacy and often need to be withdrawn due to secondary effects or pro-arrhythmia. ${ }^{3}$ Thus, ablation is the only chance for a definitive treatment in many patients. Ablation procedures require moving catheters inside the vascular system and cardiac chambers to further identify the arrhythmia substrate before ablation. Proper catheter manipulation requires a skilled electrophysiologist working under fluoroscopic guidance. This may result in significant X-ray exposure, which is a risk for both patients and medical staff. In the last two decades, different navigation systems able to create electro-anatomical maps (EAM) have led to a decrease in X-ray exposure ${ }^{4}$ and to a better understanding of the substrate of cardiac arrhythmias. ${ }^{5-8}$ However, moving and placing catheters into specific regions of the heart still requires manual guidance, which makes these procedures highly dependent on operator skills. In addition, the constant beating of the heart makes stability one of the main problems of radiofrequency delivery in certain target cardiac regions. New remote navigation systems have been recently developed with the objective of overcoming such limitations and allowing the operators to be away from the X-ray source while they are moving the catheters inside the cardiovascular system. ${ }^{9-11}$ Two remote navigation systems are currently commercially available; the robotic catheter control system (Sensei

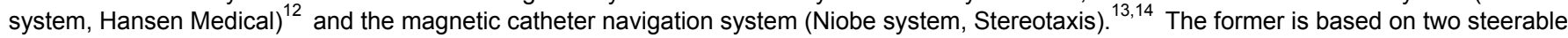
sheaths, through which any conventional catheter can be introduced for further manipulation via a pull-wire mechanism by a robotic arm fixed at a standard fluoroscopy table. The second system is based on two permanent magnets positioned on each side of the patient's body to create a uniform magnetic field. Special catheters with magnets affixed to their distal end can be navigated within the cardiac chambers by changing the orientation of the external magnetic fields. Shortcomings such as safety and similar to manual navigation or weak tissue contact force and lack of real time catheter response are present in Sensei and Niobe, respectively.

In this report, we describe the features and potential ablation capabilities of a recently developed navigation system, the Catheter Guidance, Control and Imaging $(\mathrm{CGCl}){ }^{15,16}$

\section{Protocol}

Patients who underwent ablation of a right or left atrium flutter substrate were included (Table 1). The presence of decompensated or severe systemic disease, thrombus within the left atrium, renal failure, age $<18$ years, body mass index $>40$ and pregnancy were the exclusion criteria. 
This protocol is part of the initial safety and feasibility protocol approved by the Institution Research and Ethics Committee. No atrial fibrillation procedures were included in this protocol. All patients gave informed consent.

\section{Description of the Robotic Magnetic Navigation System}

The Catheter Guidance Control and Imaging ( $\mathrm{CGCl})$ system employs eight powerful electromagnets to produce a highly agile magnetic field (to 0.16 Tesla) within an effective control area optimized to focus and contain the magnetic field almost entirely within the magnetic chamber. The magnetic chamber operates silently, has no moving parts and cools the electromagnet coils with non-toxic mineral oil. Current-regulated amplifiers provide the power to the magnetic chamber coils. The system produces magnetic fields 10 to 20 times less in intensity than Magnetic Resonance Imaging and no magnetic fields are generated when it is not in magnetic guidance mode. The magnetic field generators provide torque and force for moving, positioning and directing the tip of a catheter equipped with three permanent magnet pellets attached to its distal end.

The Robotic system includes an Operation Console, the CGCI Controller computer and a motorized linear catheter advancement mechanism. The system uses a standard 3-axis joystick, which is used to rotate the magnetic field and manually advance or retract the catheter. A 3D Controller is used to push the catheter in any screen-oriented direction. The X-Ray C-Arm may be rotated or extracted at the Operation Console using the X-Ray Dialog. The Operation Console unifies the display of the $\mathrm{CGCl}$ system, electroanatomical mapping system, EP recording system, intracardiac ultrasound (ICE), and X-Ray. It allows the direct keyboard and mouse control over the electroanatomical mapping system and EP recording system. Typically, the center screen is used for the electroanatomical mapping system screen and the CGCI overlay graphics.

\section{Patient's Preparation}

Admit the patient to the hospital the same day or the day before the procedure. Overnight fasting state is required.

1. Position the patient on the operation table, where the nursing staff cannulates a peripheral intravenous catheter before the procedure.

2. Outside of the magnetic chamber, the medical staff obtains right and left vein femoral access under local anesthesia with lidocaine. Place sheath introducers into the right and left femoral veins. We recommend guiding left atrium substrates using intracardiac echocardiography, which probe is introduced through a 9 French (Fr) left femoral vein sheath and positioned into the right atrium.

3. For left atrium substrates, administer an initial intravenous bolus and repeated doses of heparin to maintain an activated clotting time of 250-300 s.

4. Administer an initial bolus of $2 \mathrm{mg}$ of midazolam followed by continuous intravenous propofol sedation and intermittent intravenous bolus of morphine hydrochloride during the procedure.

\section{Conventional Electrophysiological Study}

1. Position standard catheters through the femoral sheath introducers into the right cardiac chambers for conventional diagnostic electrophysiological study. Use fluoroscopy guidance during manually positioning.

2. Position a decapolar catheter into the coronary sinus and a screw-in catheter in the right atrial septum.

3. Introduce a special steerable sheath with 3 electrodes along its distal end through the right femoral vein (Figure 1A) and place it in the inferior vena cava or the lower right atrium.

4. Introduce a $7 \mathrm{Fr}$ magnetized catheter into the cardiac chambers through the special sheath (Figure 1A, 1B). Next, perform conventional work up of the arrhythmia mechanism.

5. If necessary (e.g. left atrium flutter mechanism), trans-septal access to the left atrium is achieved using a trans-septal sheath and continuous ICE monitoring. For left atrial flutter procedures also place a multipolar catheter into the left atrium through the trans-septal puncture.

\section{Preparing for Remote Navigation. Catheter, Sheath, and Catheter Advancement Mechanism Assembly}

1. The motorized linear catheter advancement mechanism consists of a sterilizable wheel drive gearbox and a motor base. It also includes a disposable sheath clip and leg mount (Figure 2A). The device adds or removes catheter slack.

2. Mount the sheath in the clip and insert the catheter into the sheath, and manually advance it into the right/left atrium. The latter is confirmed by the mapping system and fluoroscopy. For left atrial substrates position the sheath in the right atrium in close proximity to the interatrial septum.

3. Next, place catheter's shaft between the rollers of the wheel drive gearbox by pulling back on the thumb lever.

4. The X-Ray C-Arm is moved to its operational position within the magnetic chamber. Patient's table is now advanced to localize the thorax within the magnetic field.

5. The operator leaves the operation room and takes control from the operation console.

6. The Magnetic catheter and the special sheath with electrodes are now shown on the center screen. All CGCl and electroanatomical mapping system operational functions are available at the CGCI operation Console.

\section{Remote Navigation and Mapping}

1. Perform catheter contact calibration of the electrical coupling index system. Maximum and minimum contact values are set up on the control panel.

2. Full integration with the electroanatomical mapping system and a closed-loop servo system allow remote navigation and 3D geometrical reconstruction of the right/left cardiac chambers, which allows the procedure to continue with minimal X-ray exposure. 
3. Use a right hand 3D Controller to steer the catheter to specific sites within the right/left atrium by changing the direction of the magnetic field (Figure 2B). A yellow magnetic arrow indicates the direction of the magnetic field. Use a left hand 3-axis joystick to control the amount of catheter slack (Figure 2C). The motorized device placed at the patient's leg allows the addition and retraction of catheter slack (Figure 2A).

4. Rapid changes in the magnetic field magnitude, direction, and gradient yield push/pull and/or torque (bend) movements in the distal portion of the catheter. Real-time remote navigation is assessed based on field vector adjustments and subsequent response in the tip of the catheter. A magnetic icon displays the coil power values as colors. Green indicates a strong positive field, and red indicates a strong negative field (Figure 3).

5. The catheter tip is aligned parallel to the vector direction of the magnetic flux density. The magnetic field gradient generated for force control of the catheter is up to $0.7 \mathrm{~T} /$ meter, with a maximal perpendicular force exerted of $25 \mathrm{~g}$.

6. Perform geometrical point acquisitions from the 4 poles of the magnetized catheter while slowly moving the catheter all around the atrial chambers. A 3D EAM reconstruction can be obtained after few minutes (Figure 3A, 3B).

7. Remote navigation with the steerable sheath positioned in the right atrium allows reaching the right inferior pulmonary vein by either directly deflecting the catheter upon crossing the interatrial septum or after making a loop in the opposite wall and then pointing the catheter towards the right inferior pulmonary vein.

8. Generate activation, voltage and first post-pacing interval maps to characterize the reentrant circuit (Figure 4A, 4B). Identify ablation targets and localize them on the 3D geometry.

\section{Ablation. Manual and Automated Catheter Positioning in the Target Sites}

1. In Automated Magnetic Guidance mode, the operator can automatically guide the catheter to specific targets by double-clicking on an electroanatomical mapping system label. This represents an essential feature to create remote and automatic ablation lines.

2. For each individual target and with the CGCl system operating in the automated mode, drive the catheter to the target points (Figure 4C). In Automated mode, the system displays a targeting dialog. This indicates the intended target, range, time and targeting search status.

3. Accuracy to automatically position the catheter on the target is also measured by the distance of the final location of the catheter to the initial manual positioning. The distance is measured in $\mathrm{mm}$ and considered significant if larger than $3 \mathrm{~mm}$. Catheter navigation accuracy requires using the field-scaling tool of the electroanatomical mapping system.

4. Manual intervention of an automatic search is possible by using the joystick or 3D Controller.

5. To terminate the arrhythmia deliver radiofrequency energy to specific sites labeled as target points, either manually or automatically guided. The arrhythmia terminates and sinus rhythm is restored upon interruption of the reentrant circuit (Figure 4C, 4D). No re-induction by fast atrial pacing confirms the elimination of the arrhythmia.

The safety of the $\mathrm{CGCl}$ system is evaluated for each one of the experimental steps.

Representative Results

This new remote magnetic navigation system allows real-time remote catheter navigation inside the right and left atrial chambers, either in the operator or automated mode. The latter is obtained after almost instantaneous field vector adjustments of direction and intensity of torque, bending, rotating, and field gradient for axial push-pull movement (See video for illustration).

The system allows arrhythmia termination upon radiofrequency delivery in the absence of major complications (cardiac tamponade, pulmonary embolism or major hemorrhages) during this initial ablation experience (Figure 4)

The automated catheter remote navigation is highly reproducible, accurate, and rapid to position and keeps the catheter tip on the desired target. Based on five initial experimental procedures the navigation was $95.7 \%$ reproducible, the average accuracy was $1.9 \pm 0.9 \mathrm{~mm}$ and the average time to reach the target was $23.28 \pm 14.8 \mathrm{sec}$. We considered nine targets in the right atrium (coronary sinus, 2 locations at the high right atrium, 3 locations at the tricuspid annulus, His, superior vena cava and inferior vena cava), nine targets in the left atrium ( 2 locations at the left atrial appendage, 3 locations at the mitral annulus and one location at each one of the pulmonary veins), six target sites in the right ventricle (2 locations at the right ventricular outflow tract, apex, right ventricle free wall, inferior wall and septum) and five more targets in the left ventricle (apex, anterior wall, lateral wall, septum and left ventricular outflow tract). Neither time, accuracy nor reproducibility to reach a target site were significantly different between chambers and targets.

\begin{tabular}{|c|c|c|c|c|c|}
\hline Type of Arrhythmia & Atrial Substrate & Type of ablation & Acute Success & \begin{tabular}{|l|} 
Procedure-derived \\
Complications
\end{tabular} & Recurrences \\
\hline \multirow[t]{3}{*}{ LA flutter $(n=3)$} & Right PVs & Posterior wall line & Yes & None & No. 6 months FU \\
\hline & Left PVs & Roof line & Yes $^{*}$ & None & No. 4 months FU \\
\hline & Inferior lateral wall & Focal RF delivery & Yes $^{* *}$ & None & No. 3 months FU \\
\hline \multirow[t]{2}{*}{ RA flutter $(\mathrm{N}=2)$} & \multirow{2}{*}{$\begin{array}{l}\text { Counterclockwise } \\
\text { cavo-tricuspidlsthmus } \\
\text { dependent RA flutter }\end{array}$} & \multirow{2}{*}{$\begin{array}{l}\text { Cavo-tricuspidlsthmus } \\
\text { line }\end{array}$} & Yes & None & No. 10 months FU \\
\hline & & & Yes & None & No. 11 months FU \\
\hline
\end{tabular}

Table 1. Atrial substrates and ablation results using the CGCI system. FU: follow up. LA: left atrium. RF: radiofrequency. RA: right atrium. PV: pulmonary vein. ${ }^{*}$ Induction of 2 more non sustained and non clinical LA flutter morphologies. ${ }^{* *}$ LA flutter no longer re-inducible. Induction of atrial fibrillation at the end of the study. ${ }^{* *}$ The presence of pericardial effusion was assessed by intracardiac echocardiography throughout the procedure. 

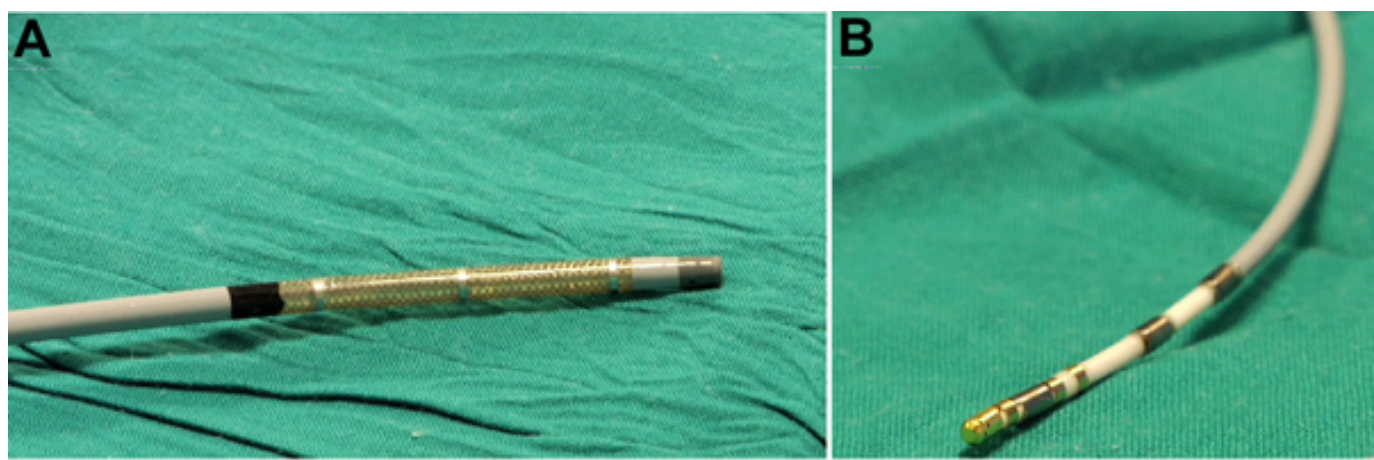

Figure 1. Agilis ES sheath (A) and MedFact magnetized and irrigated gold tip catheter (B) used for geometrical reconstruction and ablation in the right and left atrial chambers.

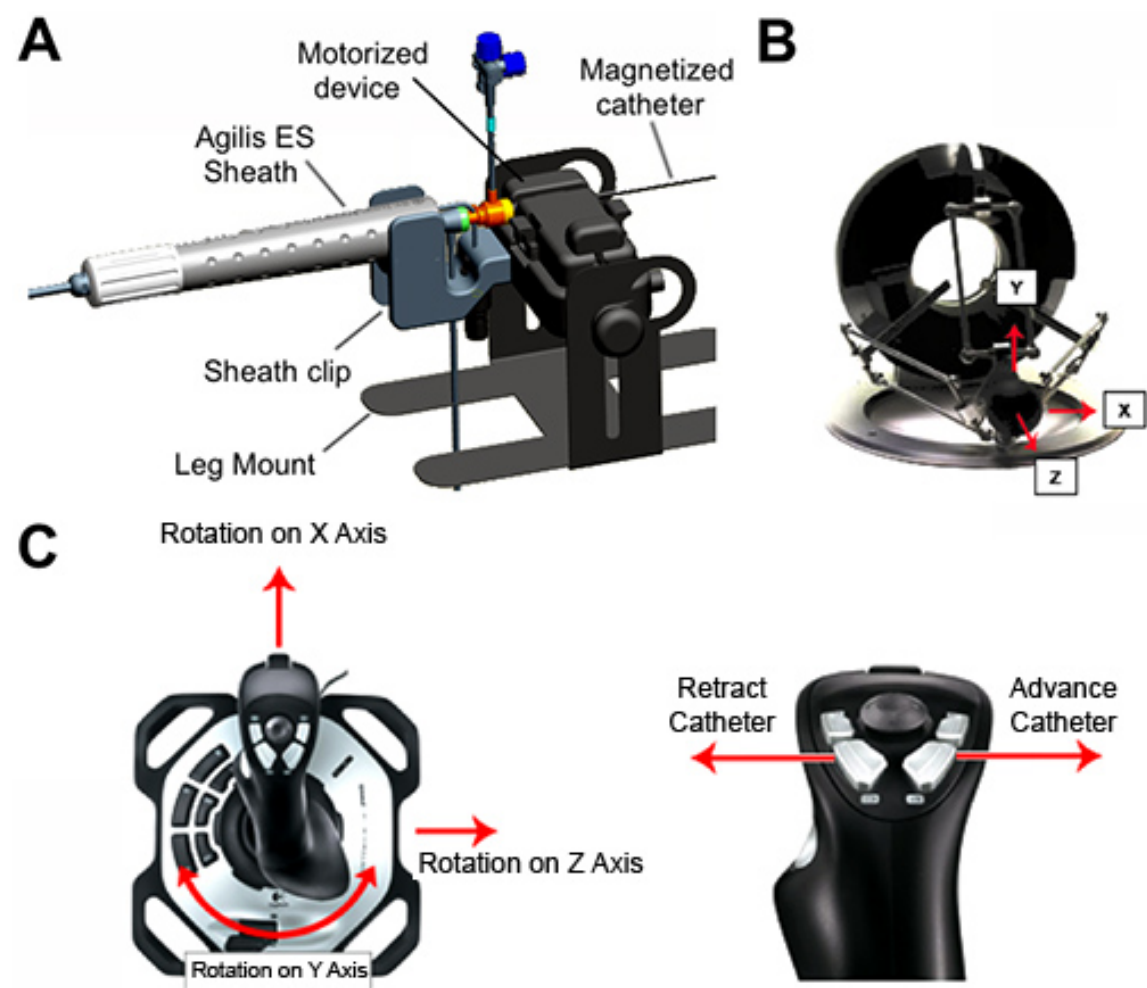

Figure 2. Components for remote navigation. A, motorized linear catheter advancement mechanism with a sterilizable wheel drive gearbox and a motor base. It includes a disposable sheath clip and leg mount. The magnetized catheter is inserted into the sheath and manually advanced into the right/left atrium. B, right hand 3D Controller used to steer the catheter to specific sites within the atrial chambers by changing the direction of the magnetic field. C, left hand 3-axis joystick to control the amount of catheter slack. 

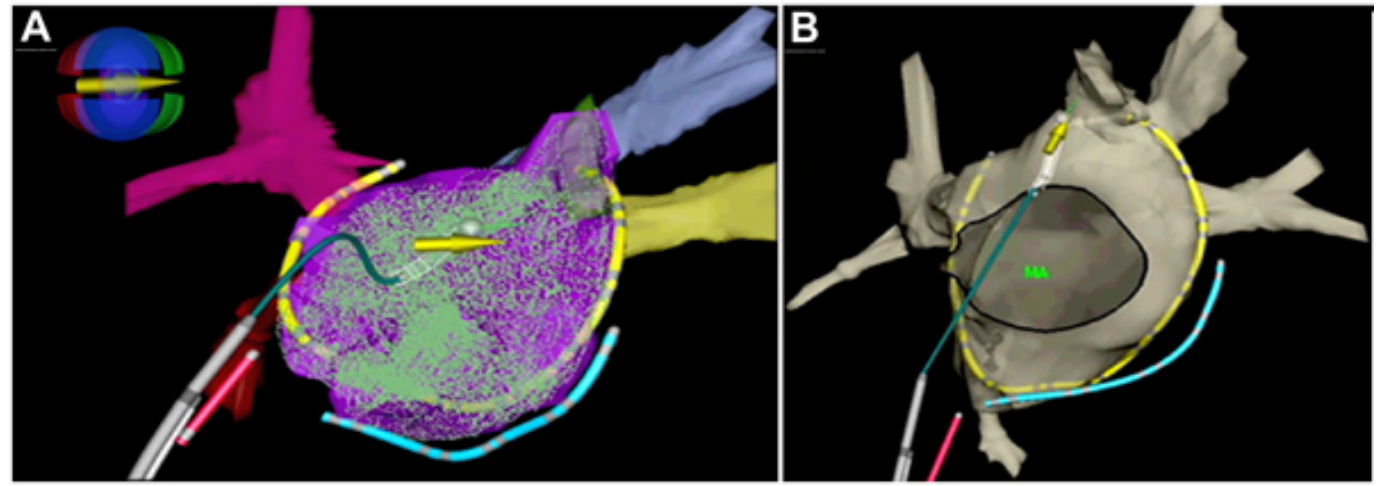

Figure 3. 3D electro-anatomical mapping geometry of the left atrium and pulmonary veins. A, geometrical point acquisitions within the left atrium using the magnetized catheter, which is represented with the white tip and the overlaying yellow arrow. The magnetic icon displays the coil power values as colors: green indicates a strong positive field, and red indicates a strong negative field. In blue a catheter into the coronary sinus. In red a screw-in catheter positioned in the right atrial septum. The multipolar catheter is shown in yellow. B, final view of the 3D anatomical reconstruction of the left atrium. MA; mitral annulus.
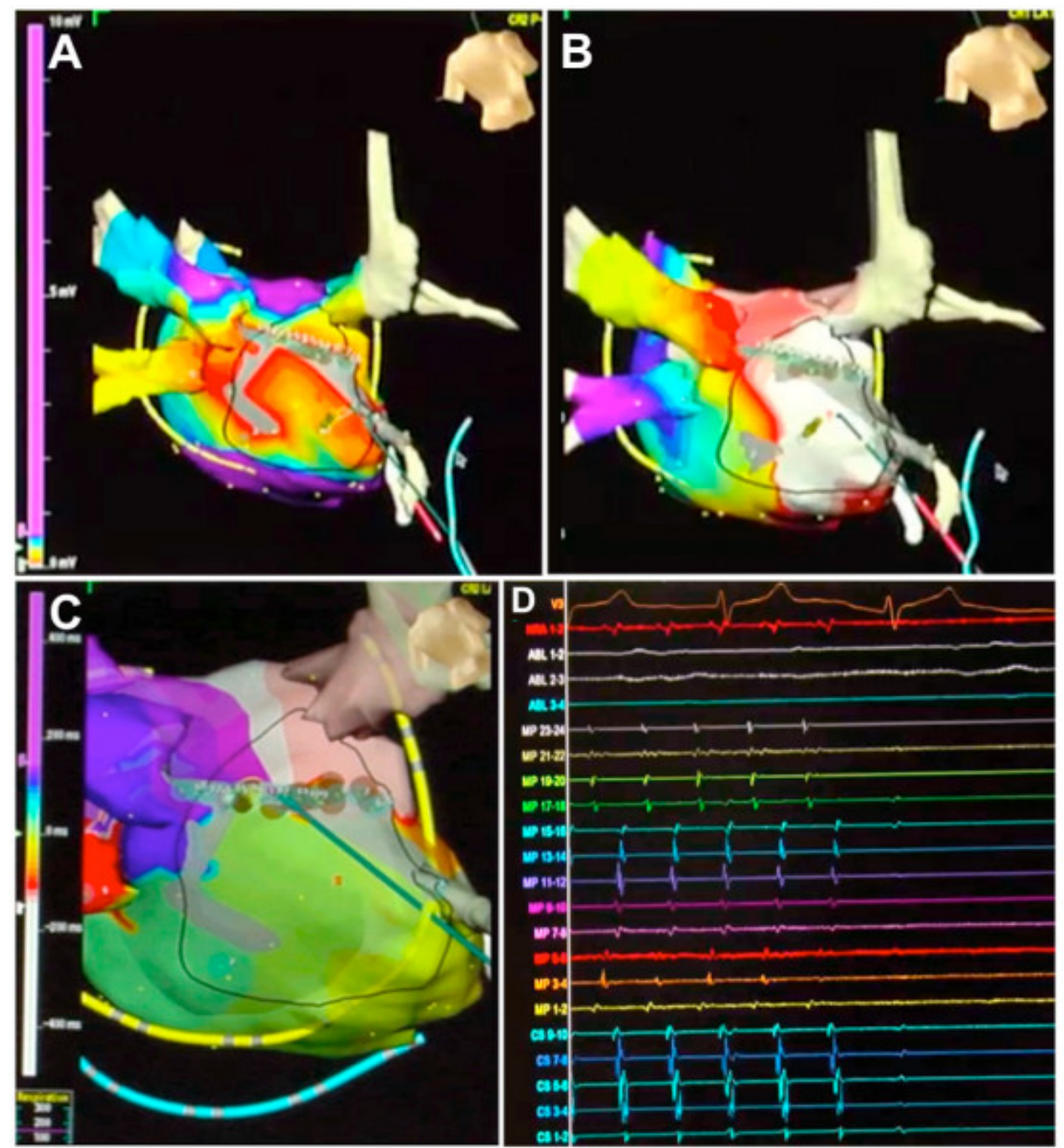

Figure 4. Interruption of a left atrial flutter circuit around the right pulmonary veins after automatically guided radiofrequency energy delivered to specific sites in the posterior wall of the left atrium. A, voltage map shows a dense scar in the posterior wall of the left atrium (grey color). B, first post-pacing interval map generated to characterize the reentrant circuit, which is localized around the right pulmonary veins (posterior view is shown). White and red indicate first post-pacing intervals $<30 \mathrm{msec}$. $\mathbf{C}$ and $\mathbf{D}$, in automated mode the catheter is driven to the target points and the arrhythmia terminates after completing a line in the posterior wall of the left atrium, which interrupts the reentrant circuit. Click here to view larger figure. 


\section{Discussion}

This is the first clinical report using the CGCI remote navigation system. It shows important technical features that might facilitate both navigation and ablation in right and left atrial substrates. The system may potentially overcome some of the limitations of the former magnetic-based Niobe system. ${ }^{10}$ Thus, endocardial contact force and navigation inside the cardiac chambers may substantially improve by increasing the strength of the magnetic field magnitude up to 0.16 Tesla compared with 0.08 Tesla in the Niobe system. Continuous and rapid shaping and reshaping of the magnetic field, rather than moving external magnets to change the magnetic field, ${ }^{13,14}$ provides instantaneously transmitted changes to the tip of the magnetized catheter leading to almost real\#time remote navigation. In automated mode the CGCl system also provides a true closed-loop servo system that has the ability to keep the catheter tip on a desired anatomic target by continuously adjusting the direction and intensity of the magnetic fields. ${ }^{17}$

The Sensei system, based on steerable sheaths manipulated within the heart by a mechanical robotic arm, allows real-time catheter movements. ${ }^{12}$ However, the use of mechanical forces to drive the catheter does not represent a technological advance over manual manipulation. Initial reports using the Sensei system had raised the concern of higher rate of cardiac tamponade than conventional manual radiofrequency delivery, ${ }^{18,19}$ which might have been related to remote manipulation of the stiff steerable Artisan catheter (Hansen Medical, Mountain View, CA, USA). Further experience and introduction of a special feature of the system to indirectly estimate catheter contact force on the tissue (IntelliSense) have shown that tamponade rates are not superior to conventional manual approach and may be more related to different temperature and power radiofrequency settings. ${ }^{20}$

The CGCl system does not require a lab with specific magnetic isolation since the magnetic field is highly focused on patient's torso. In addition, the electrophysiology room can be used either as a conventional electrophysiology lab or as a magnetic lab by moving patient's table from its regular position towards the magnetic chamber. The latter can be done either manually or by remote control. Although no major complications have been present in this initial experience, in case of serious complications, such as pericardial effusion and tamponade, it would be possible to remove the patient from the magnetic chamber in $\approx 15 \mathrm{sec}$.

General demerits such as lack of real-time contact force monitoring or lesion visualization still apply to the CGCl system. Combining robotic navigation with real-time contact force catheters and direct visualization of the atrial cavities may be a future feasible approach to increase long-term success of ablation lesions and decrease the risk of complications. To date, experimental data using the CGCl system in pigs have demonstrated reproducible navigation and accurate and rapid catheter positioning on the selected ablation targets within the atrial chambers. ${ }^{17}$ Once the ablation target is localized, the system has the ability to navigate the catheter tip to the selected target despite the cardiac motion and anatomical irregularities. Furthermore, necropsy studies in the same animals revealed that the majority of radiofrequency lesions were transmural. ${ }^{17}$ In this first report in humans the system also shows reproducible navigation and accurate and rapid catheter positioning on the selected ablation targets within the right or left atrial chambers. The use of rapid magnetic field adjustments might enhance catheter tip stability and result in fewer radiofrequency applications as well as fewer major complications. Although the results and follow up in this initial experience are encouraging, future large and randomized clinical trials in patients undergoing complex catheter-guided ablation procedures are needed to demonstrate these potential benefits.

\section{Disclosures}

Dr. José L. Merino received grants for clinical research from Magnetecs Inc. and Boston Scientific. Dr. José L. Merino served as a speaker for St. Jude Medical. Dr. Gang and Yehoshua Shachar have equity interest in Magnetecs Corp.

\section{Acknowledgements}

Supported in part by Magnetecs Inc. (Inglewood, California, USA) in collaboration with the Comunidad de Madrid and La Paz University Hospital. We thank Iván Filgueiras-Rama and Jaime Palomo-Cousido for their help in the illustration of the figures and video editing.

\section{References}

1. Evans, G.T., Jr., et al. The Percutaneous Cardiac Mapping and Ablation Registry: final summary of results. Pacing Clin. Electrophysiol. 11, 1621-1626 (1988).

2. Cappato, R., et al. Updated worldwide survey on the methods, efficacy, and safety of catheter ablation for human atrial fibrillation. Circ. Arrhythm. Electrophysiol. 3, 32-38, doi:10.1161/CIRCEP.109.859116 (2010).

3. Lafuente-Lafuente, C., Mouly, S., Longas-Tejero, M.A., Mahe, I., \& Bergmann, J.F. Antiarrhythmic drugs for maintaining sinus rhythm after cardioversion of atrial fibrillation: a systematic review of randomized controlled trials. Arch. Intern. Med. 166, 719-728, [pii] 166/7/719 doi:10.1001/archinte.166.7.719 (2006).

4. Reddy, V.Y., et al. Catheter ablation of atrial fibrillation without the use of fluoroscopy. Heart Rhythm. 7, 1644-1653, doi:10.1016/ j.hrthm.2010.07.011 (2010).

5. Knackstedt, C., Schauerte, P., \& Kirchhof, P. Electro-anatomic mapping systems in arrhythmias. Europace. 10 Suppl. 3, iii28-34, doi:10.1093/ europace/eun225 (2008).

6. Merino, J.L., Guzman, G., \& Fernandez-Cuadrado, J. Atrial fibrillation ablation guided by computed tomography. Rev. Esp. Cardiol. 62, 314, [pii] 13133308 (2009).

7. Merino, J.L., Refoyo, E., Peinado, R., \& Cuesta, E. Real-time representation of multielectrode ablation catheters by integration of computed tomographic geometry with three-dimensional electroanatomic mapping of left atrium and pulmonary veins. Heart Rhythm. 5, 628-629, [pii] S1547-5271(07)01259-3 doi:10.1016/j.hrthm.2007.12.020 (2008). 
8. Piorkowski, C., et al. Computed tomography model-based treatment of atrial fibrillation and atrial macro-re-entrant tachycardia. Europace. 10, 939-948, doi:10.1093/europace/eun147 (2008).

9. Ernst, S. Magnetic and robotic navigation for catheter ablation: "joystick ablation". J. Interv. Card. Electrophysiol. 23, 41-44, doi:10.1007/ s10840-008-9272-8 (2008).

10. Nguyen B.L., Merino J.L., \& E.S., Gang. Remote Navigation for Ablation Procedures - A New Step Forward in the Treatment of Cardiac Arrhythmias. European Cardiology. 6, 50-56 (2010).

11. Schmidt, B., et al. Remote navigation systems in electrophysiology. Europace. 10 Suppl. 3, iii57-61, [pii] eun234 doi:10.1093/europace/ eun234 (2008).

12. Al-Ahmad, A., Grossman, J.D., \& Wang, P.J. Early experience with a computerized robotically controlled catheter system. J. Interv. Card. Electrophysiol. 12 (3), 199-202, doi:10.1007/s10840-005-0325-y (2005).

13. Ray, I.B., et al. Initial experience with a novel remote-guided magnetic catheter navigation system for left ventricular scar mapping and ablation in a porcine model of healed myocardial infarction. J. Cardiovasc. Electrophysiol. 18, 520-525, [pii] JCE794 doi:10.1111/ j.1540-8167.2007.00794.x (2007).

14. Ernst, S., et al. Initial experience with remote catheter ablation using a novel magnetic navigation system: magnetic remote catheter ablation. Circulation. 109, 1472-1475, [pii] 01.CIR.0000125126.83579.1B doi:10.1161/01.CIR.0000125126.83579.1B (2004).

15. Nguyen, B.L., Farkas, L., Marx, B., et al. In: Heart Rhythm Society's 30th Annual Scientific Sessions., Boston, MA, (2009).

16. Nguyen, B.L., Merino, J.L., \& Gang, E.S. Remote Navigation for Ablation Procedures - A New Step Forward in the Treatment of Cardiac Arrhythmias. European Cardiology. 6, 50-56 (2010).

17. Gang, E.S., et al. Dynamically shaped magnetic fields: initial animal validation of a new remote electrophysiology catheter guidance and control system. Circ. Arrhythm. Electrophysiol. 4, 770-777, doi:10.1161/CIRCEP.110.959692 (2011).

18. Bernabei, M.A., Ugarte, R.C., Martin, D.T., et al. In Heart Rhythm Society's 30th Annual Scientific Sessions., Boston, MA, (2009).

19. Saliba, W., et al. Atrial fibrillation ablation using a robotic catheter remote control system: initial human experience and long-term follow-up results. J. Am. Coll. Cardiol. 51, 2407-2411, doi:10.1016/j.jacc.2008.03.027 (2008).

20. Hlivak, P., et al. Robotic navigation in catheter ablation for paroxysmal atrial fibrillation: midterm efficacy and predictors of postablation arrhythmia recurrences. J. Cardiovasc. Electrophysiol. 22, 534-540, doi:10.1111/j.1540-8167.2010.01942.x (2011). 